\title{
A Single-Arm, Prospective Study of Laser-Assisted Marsupialization for Epiglottic Cysts in Adults
}

\author{
Sangheon Park \\ Kwang-Yoon Jung \\ Min Woo Park \\ Jaewoong Hwang \\ Sei-Young Lee \\ Seung-Kuk Baek
}

Department of Otolaryngology-Head and Neck Surgery, Korea University College of Medicine, Seoul, Korea
Received November 28, 2013

Revised December 1, 2013

Accepted December 15, 2013

\section{Correspondence}

Seung-Kuk Baek

Department of Otolaryngology-Head and Neck Surgery, Korea University College of Medicine, 126-1, Anam-dong 5-ga, Seongbuk-gu, Seoul 136-705, Korea

Tel: +82-2-920-5486

Fax: +82-2-925-5233

E-mail: mdskbaek@gmail.com

(C) Korean Society for Laser Medicine and Surgery

(c) This is an open access article distributed under the terms of the Creative Commons Attribution NonCommercial License (http://creativecommons.org/ licenses/by-nc/3.0) which permits unrestricted noncommercial use, distribution, and reproduction in any medium, provided the original work is properly cited.

\section{Background and Objectives}

The aim of the current study is to estimate the feasibility of CO2 laserassisted marsupialization for epiglottic cysts under laryngeal microsurgery.

\section{Materials and Methods \\ Investigations were performed prospectively in 18 consecutive patients who had undergone laser marsupialization for epiglottic cysts. The following data as clinical factors were collected from participants: gender, age, symptoms and tumor size at presentation, pathologic data, symptom improvement, postoperative complications, and recurrence.}

\section{Results}

The study population consisted of 12 male and 16 female patients with a mean age of 55.7 years. The most common symptom was globus sensation (50\%). The mean size of the cysts was $2.7 \mathrm{~cm}$, and, histologically, 12 patients (66.7\%) had epithelial cyst, four patients (22.2\%) tonsillar cyst, and two patients $(11.1 \%)$ oncocytic cyst. There was no occurrence of significant complications or recurrence during the followup period in all patients.

\section{Conclusion}

Laser-assisted marsupialization under a laryngomicroscope for epiglottic cysts may be a useful technique compared with complete removal.

\section{Key words}

Laser; Marsupialization; Epiglottic cysts 


\section{INTRODUCTION}

Laryngeal cysts comprise approximately $5 \%$ of benign laryngeal lesion and originate from the lingual surface of epiglottis in a considerable proportion of cases. $^{1,2}$ Epiglottic cysts are thought to result from the ductal obstruction of the mucous gland and mucus retention. The epiglottic cysts may be present with a variety of clinical symptoms, including throat discomfort, sore throat, and hoarseness. However, since airway obstruction can occur by the large or infected epiglottic cyst, careful management may be important.

The conventional management of epiglottic cysts has been a complete removal of cysts. ${ }^{3}$ On the other hand, the previous study suggested that the laser marsupialization for epiglottic cyst may be useful since the cystic lesions are frequently ruptured during surgical manipulation and the complete resection of the cyst lining is difficult. ${ }^{4}$

Since there was no report about the benefit of laser assisted-marsupialization technique for epiglottic cysts in our country, we evaluated the feasibility and the effectiveness of that technique in the present study.

\section{MATERIALS AND METHODS}

Investigations were prospectively carried out in consecutive 30 patients who had undergone laser marsupialization for epiglottic cysts at the department of otolaryngology head and neck surgery, Anam Hospital, Korea University, Seoul, South Korea, between January 2004 and December 2011. Among these patients, 12 patients were excluded due to using cold instruments or Electrocautery, insufficient postoperative evaluation and management, and short and irregular follow-up period. Therefore, a total of 18 patients were included in the study. The postoperative follow-up period ranged from 3 to 40 months (mean, 10 months).

All subjectives gave informed consent to inclusion in the study. The institutional review board of our institution approved the study protocol.

\section{Surgical procedures}

All of the surgical procedures were performed under general anesthesia by the same surgeon. The cystic portion of epiglottis was completely exposed with the Steiner adjustable laryngoscope (Richard Wolf, Germany). Under an operating microscope, the cutting line was marked on the cyst roof with the operating laser, including Diode laser ( 5 W, continuous mode) or CO2 laser (250 um spot size, $5 \mathrm{~W}$, continuous mode). After the thick mucoid content within cyst was aspirated, the epiglottic mucosa covering the cyst and the connecting roof of the cyst wall were excised together. The bottom lining of the cyst was left intact.

\section{Clinical factors}

The following data as clinical factors were collected from participants: gender, age, symptoms and tumor size at presentation, pathologic data, symptom improvement, postoperative complications, and recurrence. To identify the improvement from preoperative symptom, the assessment of patients' symptoms was performed at postoperative 1 month.

\section{RESULTS}

The age at diagnosis of the 12 male and 16 female patients ranged from 44 to 76 years (mean, 55.7 years). At presentation for treatment, symptoms were as the followings: globus sensation, sore throat, and voice change. Among them, most common symptom was globus sensation (50\%). Laryngoscopic examination revealed cystic lesions with mean size $2.7 \mathrm{~cm}$ (range, 1.1-5.3 cm) (Table 1). Histologically, 12 patients (66.7\%) had epithelial cyst, 4 patients (22.2\%) tonsillar cyst, and 2 patients $(11.1 \%)$ oncocytic cyst.

Table 2 shows the results of marsupialization treatment. At postoperative 1 month, almost patients were improved without symptoms except one patient who had globus sensation. No postoperative bleeding or dyspnea was noted in any patient, except for one patient who had persistent sore throat for 1 week. In addition, No recurrence occurs during the follow-up period.

Table 1. Demographics and pathologic data of enrolled patients

\begin{tabular}{lc}
\hline \multicolumn{1}{c}{ Variables } \\
\hline Sex, no (\%) \\
Male & $12(66.7)$ \\
Female & $6(33.3)$ \\
Age, mean (range), year & $55.7(44-76)$ \\
Main symptom & \\
No & $7(38.8)$ \\
Globus sensation & $9(50)$ \\
Sore throat & $1(5.6)$ \\
Voice change & $1(5.6)$ \\
Tumor size, mean (range), cm & $2.7(1.1-5.3)$ \\
Pathologic results, no. & \\
Epithelial cyst & $12(66.7)$ \\
Tonsillar cyst & $4(22.2)$ \\
Oncocytic cyst & $2(11.1)$ \\
\hline
\end{tabular}


Table 2. Postoperative symptom improvement, complications and recurrence

\begin{tabular}{ll}
\hline \multicolumn{1}{c}{ Treatment outcome } \\
\hline Symptom improvement, no (\%) \\
Yes & $17(94.4)$ \\
No & $1(5.6)$ \\
Postoperative complication, no (\%) & \\
Bleeding & $0(0)$ \\
Sore throat* & $1(5.6)$ \\
Dyspnea & $0(0)$ \\
Recurrence, no (\%) & $0(0)$ \\
\hline
\end{tabular}

*Persistent sore throat for 1 week.

\section{DISCUSSION}

Laryngeal cysts, including epiglottic cysts, are usually benign and constitute approximately $5 \%$ of benign laryngeal lesions. ${ }^{1,5}$ The most of epiglottic cysts occur on the lingual surface of the epiglottis and show a greater prevalence in the fifth and sixth decades of life. ${ }^{6}$

The symptoms are various according to patient age, cyst size and location, airway extension. Furthermore, since the manifestation of the epiglottic cysts is nonspecific, the cyst tends to be incidentally detected in many patients. In the present study, 38.8\% of patients had no symptom associated with epiglottic cyst and the most of them were diagnosed by the procedure for other purposes, such as gastrofiberscope.

The previous study suggested that the epiglottic cysts can be histologically classified into epithelial, tonsillar, and oncocytic cysts and epithelial cyst is most common. ${ }^{7}$ The present study showed the similar proportion of cyst classification.

In the most of previous studies, complete removal of the epiglottic cysts is recommended because of the risk of recurrence. ${ }^{3,8,9}$ However, complete resection of cyst may be difficult and a time-consuming procedure, since the cyst tends to be ruptured easily during manipulation and the possibility of postoperative bleeding may increase due to an abundant vasculature of vallecula. On the other hand, laser-assisted marsupialization under laryngomicroscope may be performed easier and more rapidly, since it is possible to reduce the surgicallydissected extent by deroofing the cyst simply. Therefore, after marsupialization of cysts, rapid healing may occur between the remnant lining of the cyst and the adjacent mucosa. In addition, the wide openings of the cysts were kept without scarring or cyst recurrence during follow-up period in all patients of the present study.

In conclusion, the laser-assisted marsupialization under laryngomicroscope for epiglottic cysts showed feasibility compared with complete removal. Therefore, this technique may be appropriate to apply to the epiglottic cysts due to its advantages such as simplicity, minimal invasiveness, fast recovery, and low recurrence.

\section{REFERENCES}

1. Newman BH, Taxy JB, Laker HI. Laryngeal cysts in adults: a clinicopathologic study of 20 cases. Am J Clin Pathol 1984;81: 715-20.

2. DeSanto LW, Devine KD, Weiland LH. Cysts of the larynx-classification. Laryngoscope 1970;80:145-76.

3. Van de Water FW. Laryngeal cysts--their surgical management. Laryngoscope 1973;83:1185-94.

4. Su CY, Hsu JL. Transoral laser marsupialization of epiglottic cysts. Laryngoscope 2007;117:1153-4.

5. Lam HC, Abdullah VJ, Soo G. Epiglottic cyst. Otolaryngol Head Neck Surg 2000;122:311.

6. Su CY, Hsu JL. Transoral laser marsupialization of epiglottic cysts. Laryngoscope 2007;117:1153-4.

7. Ramesar K, Albizzati C. Laryngeal cysts: clinical relevance of a modified working classification. J Laryngol Otol 1988;102:9235.

8. Dahm MC, Panning B, Lenarz T. Acute apnea caused by an epiglottic cyst. Int J Pediatr Otorhinolaryngol 1998;42:271-6.

9. Fang TJ, Cheng KS, Li HY. A huge epiglottic cyst causing airway obstruction in an adult. Chang Gung Med J 2002;25:275-8. 